Research Paper

\title{
Effect of Comorbidity on Postoperative Survival Outcomes in Patients with Solid Cancers: A 6-Year Multicenter Study in Taiwan
}

Wen-Chi Chou ${ }^{1,2} \bowtie$, Pei-Hung Chang ${ }^{3}$, Chang-Hsien Lu ${ }^{4}$, Keng-Hao Liu ${ }^{5}$, Yu-Shin Hung ${ }^{1}$, Chia-Yen Hung1, Chien-Ting Liu', Kun-Yun Yeh³, Yung-Chang Lin'1, Ta-Sen Yeh ${ }^{5}$

1. Department of Medical Oncology, Chang Gung Memorial Hospital, Linkou, Taiwan;

2. Graduate Institute of Clinical Medical Sciences, Chang Gung University College of Medicine, Taiwan;

3. Department of Medical Oncology, Chang Gung Memorial Hospital, Chiayi, Taiwan;

4. Department of Medical Oncology, Chang Gung Memorial Hospital, Keelung, Taiwan;

5. Department of Surgery, Chang Gung Memorial Hospital, Linkou, Taiwan;

6. Department of Medical Oncology, Chang Gung Memorial Hospital, Kaohsiung, Taiwan.

$\triangle$ Corresponding author: Wen-Chi Chou, M.D. Department of Medical Oncology, Chang Gung Memorial Hospital, Linkou, 5 Fu-Hsing Street, Kwei-Shan Shiang, Taoyuan 333, Taiwan. Tel: 886-3281200 Ext: 2517 Fax: 886-3-3285818 E-mail: f12986@cgmh.org.tw or wenchi3992@yahoo.com.tw.

(C) Ivyspring International Publisher. Reproduction is permitted for personal, noncommercial use, provided that the article is in whole, unmodified, and properly cited. See http://ivyspring.com/terms for terms and conditions.

Received: 2015.12.22; Accepted: 2016.03.15; Published: 2016.04.28

\begin{abstract}
Purpose: Patients with comorbidities are more likely to experience treatment-related toxicities and death. Our aim was to examine the effect of comorbidity on postoperative survival outcomes in patients with solid cancers.

Methods: In total, 37,288 patients who underwent potentially curative operations for solid cancers at four affiliated hospitals of the Chang Gung Memorial Hospital, between 2007 and 2012, were stratified according to the Charlson Comorbidity Index (CCl) for postoperative survival analysis. Multivariate Cox regression was used to adjust hazard ratios of survival outcomes among different $\mathrm{CCl}$ subgroups.

Results: A significantly greater proportion of patients with comorbidities presented with poorer clinicopathological characteristics compared to those without. After cancer surgery, $26 \%$ of patients died after a median follow-up duration of 38.9 months. Overall mortality rates of patients with $\mathrm{CCl}$ scores of $0,1,2,3,4$, and $5-8$ were $22.9 \%, 29.5 \%, 38.2 \%, 43.2 \%, 50.2 \%$, and $56.4 \%$, respectively. After adjusting for other clinicopathological factors, patients with increasing $\mathrm{CCl}$ scores were associated with significantly reduced overall and noncancer-specific survival rates, while only patients with $\mathrm{CCl}$ scores of $>2$ were associated with higher cancer-specific mortality rates.

Conclusions: Patients with increasing numbers of comorbidities were associated with reduced postoperative survival outcomes. Patients with multiple comorbidities were most vulnerable to both cancer- and noncancer-specific deaths in the first 6 months after cancer surgery. Our results suggest that for both the patient and clinician, it should be taken into consideration about cancer surgery when dealing with multiple comorbidities.
\end{abstract}

Key words: comorbidity, Charlson Comorbidity Index, postoperative mortality, cancer surger.

\section{Introduction}

Comorbidity indicates the presence of one or more additional medical disorders co-occurring with a primary disease within the same individual. The prevalence of disease rises with age; therefore, comorbidity becomes increasingly more common over a person's life span. With the aging of the general 
population and advancements in medical care, the prevalence of chronic diseases has doubled between 1985 and 2005, and the proportion of patients with $\geq 4$ chronic diseases has increased 3-fold [1]. A large cross-sectional study from the Scotland Medical Database reported in 2007 that $42 \%$ of all patients had at least one comorbidity, while $23 \%$ had $>2$ comorbidities [2].

Comorbidity is not only a feature of old age, but is also particularly associated with people who experience poor social support, high levels of socio-economic deprivation, and mental health disorders [2]. In addition, comorbidity is often associated with a decline in functional reserves and an increasing prevalence of frailty. Therefore, patients with comorbidities are more likely to have suboptimal outcomes and experience more treatment-related toxicities. The impact of comorbidity is undoubtedly apparent in cancer patients. Previous studies have suggested that cancer patients with multiple comorbidities are associated with a delay in cancer diagnosis [3], increased risk of surgical complications $[4,5]$, higher postoperative mortality rates [6-8], and a greater usage of medical resources [9]. For example, a population-based cohort study from Sweden demonstrated that patients with comorbidities have an increased risk of all-cause mortality after curative surgery for esophageal cancer of 1.24-fold compared to those patients without comorbidities [10]. Furthermore, a review study found that patients with comorbidities have poorer survival outcomes (5-year mortality hazard ratios of 1.1-5.8 across various types of cancer) than those without comorbidities [11]. Unfortunately, in spite of the knowledge of the importance of comorbidities in cancer patients, it remains unclear whether the negative influence of comorbidity is attributable to cancer- or noncancer-specific mortality rates.

Cancer-related mortality has been recognized as the leading cause of death in Taiwan since the National Registry of Death database was made available in 1981[12, 13]. The proportion of cancer-related deaths has increased annually, accounting for $25 \%$ of the total number of deaths in Taiwan in $2014[12,13]$. The population older than 70-years of age in Taiwan has increased 2.4-fold between 1989 and 2014 [14]. With the aging of the general population and increasing incidence of cancer worldwide, there is an urgent need to improve our clinical understanding of the influence of comorbidity on postoperative outcomes in cancer patients. The aim of this study, therefore, was to examine the effect of comorbidity on survival outcomes, in particular cancer- and noncancer-specific survival rates, after surgery in patients with solid cancers.

\section{Methods}

\section{Patient selection}

In total, 37,288 patients who underwent radical surgery for solid cancers at four affiliated hospitals of the Chang Gung Memorial Hospital (Linkou, Keelung, Chiayi, and Kaohsiung) between January 2007 and December 2012 were included in this study. Patients with pathologically or radiographically suspicious malignancies underwent radical resection of their primary cancers with curative intent. Patients who underwent palliative resection or bypass surgery were excluded, as were patients with skin cancers and superficial urinary bladder cancers. All patients were stratified into subgroups, according to comorbidity using the Charlson Comorbidity Index (CCI) [15], for postoperative survival analysis. This study was approved by the Institutional Review Boards at all four affiliated hospitals of the Chang Gung Memorial Hospital, in compliance with the Helsinki Declaration fourth revision (1996).

Table 1. Scoring of Charlson comorbidity index ${ }^{15}$

Comorbidity component (apply 1 point to each unless otherwise note)

Myocardial infarction

Congestive heart failure

Peripheral vascular disease

Cerebrovascular disease

Dementia

Chronic obstructive pulmonary disease

Connective tissue disease

Peptic ulcer disease

Diabetes mellitus (2 points if end-organ damage)

Moderate to severe chronic kidney disease (2 points)

Hemiplegia (2 points)

Leukemia or lymphoma (2 points)

Solid cancer ( 2 points, 6 points if metastatic

Liver disease ( 3 points if moderate to severe)

Acquired immune deficiency syndrome ( 6 points)

Charlson comorbidity index $(\mathrm{CCI})=$ sum of points of comorbidity component. A modified CCI that excluding the scores for solid cancer was used in this study.

\section{Data collection}

Administrative and clinical data collected before cancer surgery included patient demographics (age, gender, Eastern Cooperative Oncology Group [ECOG] performance status, admission mode [elective or emergency], prior history of cancer, cancer site by anatomical location, histological grade of tumor differentiation, and clinical tumor stage), American Society of Anesthesiologist (ASA) physical status, and CCI scores. Demographic information was recorded by primary care clinicians using a prospectively formulated electronic record form provided by the institutional Cancer Center with the intent of improving the quality of care for cancer patients in Taiwan since the implementation of the 
2006 Cancer Prevention and Treatment Act. Data quality was maintained for completeness and accuracy by individual multidisciplinary teams and well-trained Cancer Center personnel. Tumor stage was recorded as localized, regional, advanced, and unclassified using the Surveillance, Epidemiology, and End Results Program summary stage classification [16]. ASA scores were provided at preanesthetic evaluation by anesthesiologists while CCI scores were calculated from tabulated electronic record forms using the International Classification of Diseases Ninth Revision coding. A modified CCI that excluded scores for patient age and type of cancer was used in this study. Patients with a diagnosis of more than one tumor within the study period were analyzed from the date of surgery of the first tumor. Patients receiving multiple surgeries for their primary tumors within the study period were analyzed from the date of the first surgery. All included patients were followed-up until death or June 30, 2014. Overall survival was determined from the time of surgery to any cause of death or the date last known to be alive. Cancer- and noncancer-specific mortalities were defined as death due to cancer and any cause of death other than cancer, respectively. All dates of death were obtained from either the Institutional Cancer Registry or Taiwan Cancer Registry.

\section{Statistical analysis}

Basic demographic data were summarized as $n$ (\%) for categorical variables. Differences in the distribution of clinical variables among different age groups were compared using the Chi-squared test. Survival durations were calculated using the Kaplan-Meier method. The effect of CCI scores on survival was assessed using the Cox proportional hazards model. Adjusted hazard ratios for different CCI subgroups were estimated by performing a multivariate Cox regression after adjusting for gender, age, prior history of cancer, ECOG scale, ASA score, admission mode, primary cancer site, tumor stage, and tumor grade. These nine clinicopathological variables were selected, according to our previous study [17], with the intent of minimizing potential confounding effects on postoperative survival outcome in cancer patients. Statistical analyses were conducted in Statistical Package for the Social Sciences (SPSS) for Windows Software Version 17.0 (SPSS Inc., Chicago, IL, USA). All statistical assessments were two-sided. Probability (p)-values below 0.05 were considered statistically significant.

\section{Results}

Baseline characteristics of the patients included in this study are summarized in Table 2. Among the 37,288 patients who underwent cancer surgery between 2007 and 2012, 69.1\%, 21.4\%, 6.4\%, 1.8\%, $0.8 \%$, and $0.4 \%$ exhibited CCI scores of $0,1,2,3,4$, and $5-8$, respectively. A significantly greater proportion of patients with comorbidities were male who presented with older age, greater admission from emergency departments, poorer ECOG scales, higher ASA scores, more extensive histories of cancer, more poorly differentiated grades of tumor, and greater numbers of primary tumor sites within intra-abdominal organs as compared to those patients without comorbidities.

After a median follow-up duration of 38.9 (interquartile range, 22.8-60.4) months, 9,704 patients $(26.0 \%)$ had died after cancer surgery at the date of study censor. Cancer- and noncancer-specific mortality rates were $17.6 \%$ and $8.5 \%$, respectively. Overall mortality rates of patients with CCI scores of $0,1,2,3,4$, and $5-8$ were $22.9 \%, 29.5 \%, 38.2 \%, 43.2 \%$, $50.2 \%$, and $56.4 \%$, respectively (Figure 1). Figure 2 represents a histogram of the cancer- and noncancer-specific mortality rates for each CCI subgroup at the date of study censor. In general, patients with higher CCI scores were associated with both increased cancer- and noncancer-specific mortality rates. Hazard ratios of univariate and multivariate analyses of overall, cancer-, and noncancer-specific mortality rates, as stratified according to CCI subgroups, are shown in Table 3. Patients with increasing CCI scores were associated with significantly poorer outcomes in terms of overall and noncancer-specific mortality rates, in both the univariate and multivariate analyses. Consideration of the cancer-specific mortality rates, however, revealed patients with increasing CCI scores to be significantly associated with higher mortality rates in the univariate analysis, but only patients with CCI scores of $>2$ were found to be significantly associated with higher mortality rates in the multivariate analysis compared to those patients without comorbidities.

Given many patients died within the first 2-years after cancer surgery, we calculated the mortality rates according to CCI stratification within each quarter up to the end of the first 2-years after cancer surgery, with the intent of analyzing the impact of CCI subgroups on cancer- and noncancer-specific mortality risk (Figure 3). The cancer-specific mortality rates in each quarter were consistent (range, $1.7 \%-2.0 \%$ ) during the first year after cancer surgery, and gradually declined from $1.5 \%$ in the fifth quarter to $1.1 \%$ in the eighth quarter after cancer surgery, in the overall population. By contrast, the overall noncancer-specific mortality rates were shown to be consistent (range, $0.6 \%-0.9 \%$ ) in each quarter up to the 
end of the first 2-years after cancer surgery. In general, patients with increasing CCI scores were associated with higher cancer-specific mortality rates compared to those patients without comorbidities. However, differences in cancer-specific mortality rates between different CCI subgroups were diminished until the sixth quarter after cancer surgery. Again, patients with CCI scores of $>2$ were associated with higher noncancer-specific mortality rates after cancer surgery. However, differences in noncancer-specific mortality rates between patients with CCI scores of $0-2$ and $>2$ were only observed in the first two quarters after cancer surgery.

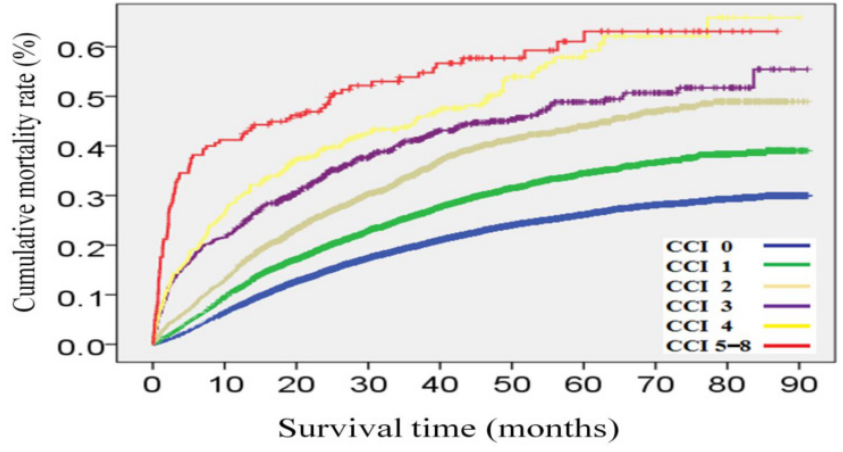

Figure 1. Cumulative mortality rates after cancer surgery stratified by Charlson Comorbidity Index (CCl) subgroups.

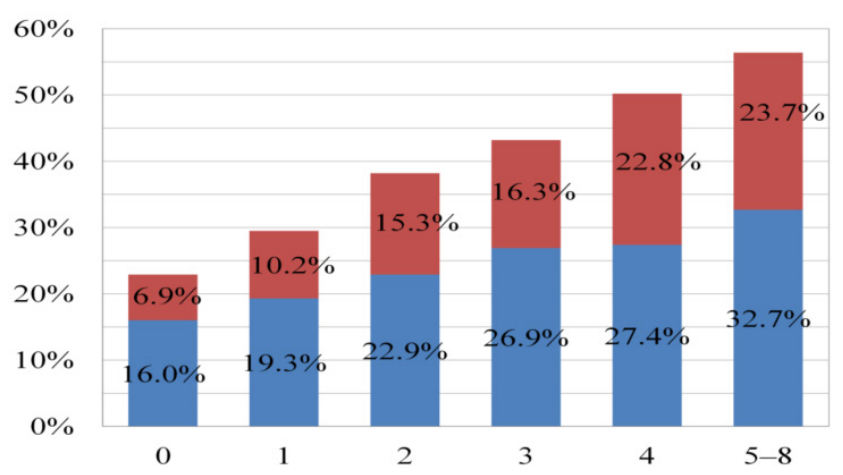

Figure 2. Histogram of overall (blue + red color), cancer-specific (blue color), and noncancer-specific (red color) mortality rates for each Charlson Comorbidity Index $(\mathrm{CCl})$ subgroup at the date of study censor.

Table 2. Basic patient characteristics stratified according to Charlson Comorbidity Index (CCl) subgroups.

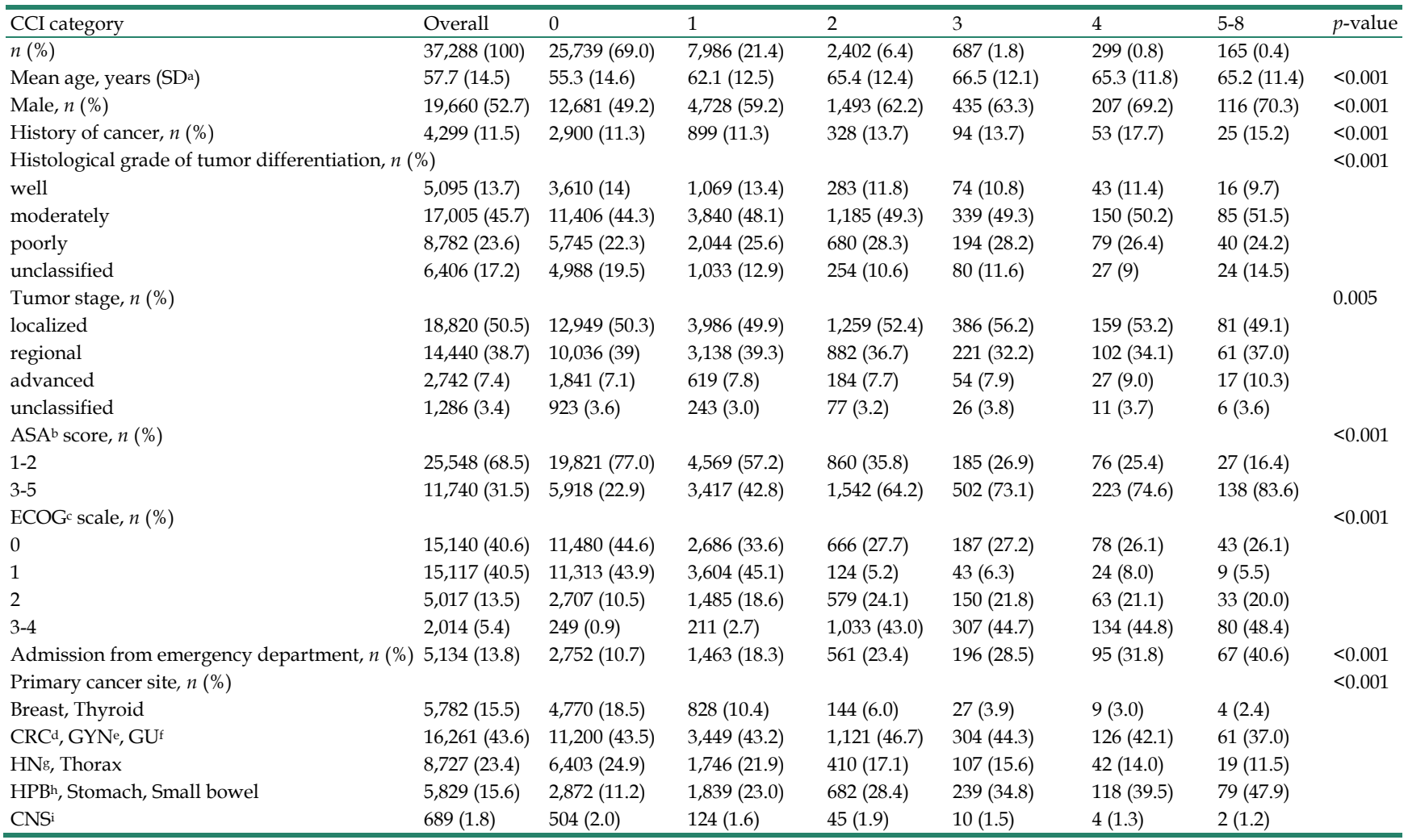

aStandard deviation; ${ }^{\mathrm{b}}$ American Society of Anesthesiologist; ${ }^{\mathrm{C} E a s t e r n}$ Cooperative Oncology Group; ${ }^{\mathrm{d} C o l o r e c t u m ;}{ }^{\mathrm{e}} \mathrm{Gynecological;}{ }^{\mathrm{f} G e n i t o u r o l o g i c a l ~} \mathrm{sHead}$ and neck; ${ }^{\mathrm{h} H e p a t o}$ pancreatico biliary; ${ }^{i}$ Central nervous system. 
Table 3. Unadjusted and adjusted analyses of causes of death stratified according to Charlson Comorbidity Index (CCl) subgroups.

\begin{tabular}{|c|c|c|c|c|c|c|c|c|}
\hline \multirow{2}{*}{$\begin{array}{l}\text { CCI } \\
\text { category }\end{array}$} & \multirow{2}{*}{$\begin{array}{l}\text { Death/total } \\
\text { patient, } \mathrm{n}(\%)\end{array}$} & \multirow{2}{*}{$\begin{array}{l}\text { Median OS, } \\
\text { months }(95 \% \mathrm{CI})\end{array}$} & \multicolumn{2}{|l|}{ Overall mortality } & \multicolumn{2}{|c|}{ Cancer-specific mortality } & \multicolumn{2}{|c|}{ Noncancer-specific mortality } \\
\hline & & & $\mathrm{HR}(95 \% \mathrm{CI})$ & AHR $(95 \%$ CI) & HR (95\% CI) & AHR(95\% CI) & HR (95\% CI) & AHR(95\% CI) \\
\hline 0 & $5,888 / 25,749(22.9)$ & NR & 1 (reference) & 1 & 1 & 1 & 1 & 1 \\
\hline 1 & $2,358 / 7,986(29.5)$ & NR & $1.38(1.32-1.45)^{c}$ & $1.05(1.00-1.11)^{\mathrm{a}}$ & $1.29(1.21-1.36)^{c}$ & $0.99(0.94-1.06)$ & $1.62(1.49-1.76)^{c}$ & $1.20(1.10-1.31)$ \\
\hline 2 & $918 / 2,402(38.2)$ & NR & $1.94(1.81-2.08)^{c}$ & $1.12(1.03-1.21)^{\mathrm{b}}$ & $1.64(1.50-1.79)^{c}$ & 0.97 (0.87-1.08) & $2.64(2.36-2.96)^{c}$ & $1.44(1.26-1.64)$ \\
\hline 3 & $297 / 687(43.2)$ & $65.4(49.1-81.7)$ & $2.46(2.19-2.77)^{c}$ & $1.21(1.07-1.37)^{c}$ & $2.16(1.87-2.51)^{c}$ & $1.32(1.12-1.54)^{b}$ & $3.18(2.63-3.85)^{c}$ & $1.76(1.43-2.16)$ \\
\hline 4 & $150 / 299(50.2)$ & $46.7(36.8-56.6)$ & $3.08(2.62-3.63)^{c}$ & $1.43(1.20-1.69)^{c}$ & $2.36(1.90-2.94)^{c}$ & $1.47(1.18-1.81)^{c}$ & $4.85(3.81-6.18) \mathrm{c}$ & $2.36(1.83-3.05)$ \\
\hline $5-8$ & 93/165 (56.4) & $25.0(8.6-41.4)$ & $4.12(3.36-5.06)^{c}$ & $2.22(1.79-2.74)^{c}$ & $3.37(2.56-4.41)^{c}$ & $1.82(1.38-2.4)^{c}$ & $5.93(4.32-8.14)^{c}$ & $3.11(2.24-4.31)$ \\
\hline
\end{tabular}

OS: overall survival; CI: Confidence interval; NR: Not reached; HR: hazard ratio; AHR: adjusted hazard ratio, ${ }^{a} \mathrm{p}<0.05,{ }^{\mathrm{b}} \mathrm{p}<0.01, \mathrm{c} p<0.001$.

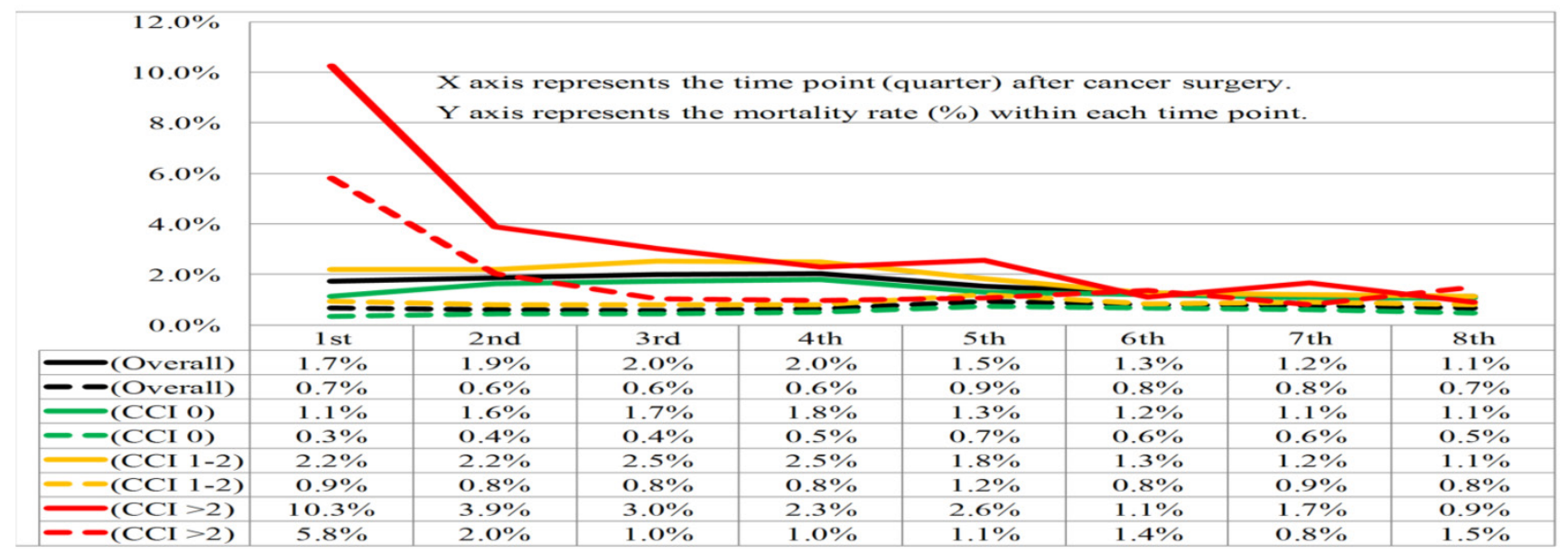

Figure 3. Cancer-specific (solid line) and noncancer-specific (dashed line) mortality rates within each quarter of the first 2-years after cancer surgery stratified by overall population (black color) and Charlson Comorbidity Index ( $\mathrm{CCl}$ ) subgroups 0 (green color), 1-2 (orange color), and >2 (red color).

\section{Discussion}

In this study, we evaluated the influence of comorbidity on survival outcomes in patients with solid cancers undergoing potentially curative resections in multiple centers within a 6-year period. Through the integration of prospectively collected electronic medical records [17] and deceased data obtained from the Taiwan Cancer Registry, we were able to analyze patient demographics, cancer types, histories of previous cancers, tumor stages, admission types, and associated medical comorbidities in a non-biased and quantitative manner with minimal absent data. Accordingly, our study identified that patients with increasing CCI scores were associated with a greater postoperative mortality risk, including increased overall, cancer-, and noncancer-specific mortality rates. Most importantly, our study also demonstrated that patients with multiple comorbidities (especially CCI scores of $>2$ ) were most vulnerable to noncancer-related deaths within the first 6 months after cancer surgery.

Surgical resection is the most common curative modality for patients with localized solid cancers. However, surgery can result in substantial morbidity and mortality in cancer patients, especially in those who are medically unfit or frail to begin with [18-22].
Consistent with previously published reports, our findings have demonstrated that patients with increasing numbers of comorbidities are associated with poorer postoperative survival outcomes, both in terms of cancer- and noncancer-specific mortality rates. However, differences in survival outcomes among patients within different CCI subgroups were less distinct after adjusting for other clinicopathological characteristics. Our results remind us that patients with comorbidities are associated with a higher prevalence of poorer clinicopathological features; therefore, the true impact of comorbidities on survival outcomes may be more predictable after adjustment or concurrent consideration of those negative predictors. For example, a recent study of ours has demonstrated that surgical mortality risk was highest among older patients with multiple concomitant comorbidities and functional limitations (50\% postoperative 3 month mortality rate compared to a $1 \%$ postoperative 3 month mortality rate in patients with no comorbidities and normal functional status [23]). The high risk of postoperative mortality for extremely frail patients should be weighed against the potential benefits in order to determine their eligibility for surgery that may assist in the decision-making process as well as the design of alternative treatment options [24]. 
Previous studies have suggested that the presence of comorbidities increases the risk of all-cause mortality in patients with cancer [25-28]. Survival outcomes in cancer patients worsen as the number of comorbidities increases [28]. The impact of comorbidity on survival outcomes in cancer patients is undoubtedly apparent, through increasing postoperative complications $[4,5]$ and poor adherence to adjuvant treatments [29-31]. Beyond inadequate treatments for cancer, several studies have suggested that cancer survivorship is associated with an increased likelihood of substandard care across a broad range of chronic medical conditions [32, 33]. In contrast, a survey that compared primary care physicians and oncologists, with respect to their practices for the care of breast and colon cancer survivors, reported that primary care physicians were less likely than oncologists to endorse routine use of recommended tests for detecting cancer recurrence [34]. Therefore, a shared care model by coordination between the oncologist and primary care physician may offer an innovative approach for the monitoring of cancer survivors $[35,36]$.

Traditionally, postoperative 30-90 day mortality rates have been used as a measurement for predicting surgical risk [37]. However, surgical complications are not limited to the immediate postoperative period. A significant number of patients live for longer than 30 days after surgery but die within a year [38]. The increment in postoperative one-year mortality risk was especially prominent in elderly patients, those with comorbidities, and those who received emergent surgeries [18]. Consistent with these findings, our study showed a steady proportion of patients dying due to cancer in each quarter during the first year after cancer surgery. Furthermore, our study also demonstrated that patients with CCI scores of $>2$ were most vulnerable to noncancer-related deaths within the first 6 months after cancer surgery. There were no significant differences in noncancer-specific mortality rates between patients of different CCI subgroups, who lived beyond 6 months after cancer surgery. Discrepancies between mortality rates at different postoperative time points, among patients with multiple comorbidities, highlights the importance of identifying patients at increased risk of death after surgery and refinement of postoperative care in cancer patients with multiple comorbidities.

In addition to well-known clinical features, including older age, poorer ECOG scales, and higher ASA scores among patients with increasing CCI scores, our study has also demonstrated that these patients are characterized by higher proportions of male gender, poorly differentiated grades of tumor, emergency department admissions, advanced tumor stages, and primary tumor sites within the stomach and hepato-biliary-pancreatic tract. In a report studied over 1.5 million people from Scotland reported that the prevalence of multimorbidity increased with age [2]. The association of male gender and poorly differentiated grade of patients with increasing CCI scores in our study possible second to higher proportion of advanced tumor stage and visceral cancer types in these patients group. Previous studies reported that patients with increasing comorbidities are particularly associated with lower socioeconomic status [2] and advanced stage [3], which often resulted in suboptimal cancer screening, treatment and follow-up. Fleming et al found women with cardiovascular and musculoskeletal diseases to be at a lower risk of receiving a diagnosis of advanced breast cancer [3]. One possible explanation is that patients with certain comorbidities (e.g., heart/lung diseases) are associated with a higher utilization of screening mammograms and greater likelihood of diagnosis of early stage breast cancer [39]. Since there is a distinct lack of specific clinical presentations and effective screening methods for the early diagnosis of visceral cancers, these reasons may possibly explain the disparities between the basic characteristics of patients with and without comorbidities in our study.

Following the installment of Cancer Prevention and Treatment Arc in Taiwan in 2006, all newly diagnosed cancer cases are reviewed by a cancer-specific multi-disciplinary team before any antitumor treatment in our institution. At Chang Gung Memorial Hospital, all patients with previous known diseases, the primary care physician will review the patient's medical record, medication and blood works to determine the status of the disease. For those found of comorbidities along with cancer diagnosis, a consultation of each specialist will be obtained as part of the preoperative evaluation and followed at an as-needed basis during the perioperative period. The goal of treating other comorbidities during the perioperative period is to prepare the patient for cancer surgery and reduce surgical risks. Once the patient is ready to be discharged, follow-up appointment is usually made to visit the specialist alongside with post-operative follow-up at an outpatient setting. If a patient preferred to find another specialist at his/her own discretion, a summary of treatment during hospitalization would be given. The patient is advised to bring his/her current medication record at each follow-up. Because the easily accessible healthcare system, in Taiwan, a patient is often followed by a specialist of each specific disease.

The strength of our study was the large patient numbers included in the design from across multiple 
institutes in Taiwan over a 6-year period. Our study evaluated the impact of comorbidities both on cancerand noncancer-specific survival outcomes after cancer surgery. Additionally, several important clinicopathological variables that can potentially influence postoperative outcomes were prospectively identified and adjusted for to minimize their potential confounding effects on survival analysis. Therefore, our study is capable of representing the true impact of comorbidity on survival outcomes after cancer surgery. However, there were some limitations. Firstly, our study only included patients who underwent cancer surgery, and there was a selection bias towards patients who were offered and had received surgical treatment. Therefore, the true postoperative mortality risks may be underestimated in patients with solid cancers and comorbidities. Secondly, since this study calculated CCI scores from medical records using the International Classification of Diseases Ninth Revision coding it was not possible to evaluate the relationship between the severity of medical illnesses and survival outcomes. Thirdly, due to the heterogeneity of different cancer types, pathological variables, and differences in postoperative organ reserves, we did not include postoperative treatments (e.g., adjuvant chemotherapy or radiation therapy) in our statistical analysis; as such, treatment options were less available to patients with multiple comorbidities and might have had a confounding effect on survival outcome.

In conclusion, our study has shown that patients with increasing numbers of comorbidities are associated with poorer survival outcomes in terms of overall, cancer-, and noncancer-specific survival rates in patients with solid cancers who underwent cancer surgery. Patients with multiple comorbidities (especially CCI scores of $>2$ ) were most vulnerable to both cancer- and noncancer-specific deaths within the first 6 months after cancer surgery. Our results suggest that for both the patient and clinician, it should be taken into consideration about cancer surgery when dealing with multiple comorbidities.

\section{Compliance with ethical standards}

Research involving human participants: All procedures performed in studies involving human participants were in accordance with the ethical standards of the institutional and/or national research committee and with the 1964 Helsinki declaration and its later amendments or comparable ethical standards.

Informed consent: For this type of study formal consent is not required.

\section{Acknowledgments}

The authors wish to thank all members of the Cancer Center at Chang Gung Memorial Hospital for their assistance in data collection.

\section{Competing Interests}

The authors have declared that no competing interest exists.

\section{References}

1. Uijen AA, van de Lisdonk EH. Multimorbidity in primary care: prevalence and trend over the last 20 years. Eur J Gen Pract. 2008; 14: 28-32.

2. Barnett K, Mercer SW, Norbury M, et al. Epidemiology of multimorbidity and implications for health care, research, and medical education: a cross-sectional study. Lancet. 2012; 380: 37-43.

3. Fleming ST, Pursley HG, Newman B, et al. Comorbidity as a predictor of stage of illness for patients with breast cancer. Med Care. 2005; 43: 132-40.

4. Backemar L, Lagergren $\mathrm{P}, \mathrm{Djärv} \mathrm{T}$, et al. Comorbidities and risk of complications after surgery for esophageal cancer: a nationwide cohort study in Sweden. World J Surg. 2015; 39: 2282-8.

5. Hamakawa T, Kurokawa Y, Mikami J, et al. Risk factors for postoperative complications after gastrectomy in gastric cancer patients with comorbidities. Surg Today. 2015; doi: 10.1007/s00595-015-1175-6.

6. Kim J, Kim S, Albergotti WG, et al. Selection of ideal candidates for surgical salvage of head and neck squamous cell carcinoma: effect of the Charlson-age comorbidity index and oncologic characteristics on 1-year survival and hospital course. JAMA Otolaryngol Head Neck Surg. 2015; doi: 10.1001/jamaoto.2015.2158

7. Melvan JN, Sancheti MS, Gillespie T, et al. Nonclinical factors associated with 30-day mortality after lung cancer resection: an analysis of 215,000 patients using the national cancer data base. J Am Coll Surg. 2015; 221: 550-63.

8. Datema FR, Ferrier MB, van der Schroeff MP, et al . Impact of comorbidity on short-term mortality and overall survival of head and neck cancer patients. Head Neck. 2010; 32:728-36.

9. Yoon SJ, Kim EJ, Seo HJ, et al. The association between Charlson comorbidity index and the medical care cost of cancer: a retrospective study. Biomed Res Int. 2015:259341.

10. Backemar L, Lagergren $\mathrm{P}$, Johar A, et al. Impact of co-morbidity on mortality after oesophageal cancer surgery. Br J Surg. 2015; 102: 1097-105.

11. Søgaard M, Thomsen RW, Bossen KS, et al. The impact of comorbidity on cancer survival: a review. Clin Epidemiol. 2013; 5: 3-29.

12. [Internet] Cancer Statistics 2014. Taiwan Cancer Registry. http://tcr.cph.edu.tw. Accessed 15 August 2015

13. [Internet] Statistics of Causes of Death 2014. Ministry of Health and Welfare, Executive Yuan, Republic of China, 2015. http://mohw.gov.tw/cht/ DOS/Statistic.aspx?f_list_no $=312 \&$ fod_list_no $=5488$. Accessed 15 August 2015

14. [Internet] Statistical Yearbook of Interior 2014. Ministry of the Interior, Executive Yuan, Republic of China, 2015. http://sowf.moi.gov.tw/stat/year/list. Accessed 15 August 2015

15. Newschaffer CJ, Bush TL, Penberthy LE, Bellantoni M, Helzlsour K, Diener-West M. Does comorbid disease interact with cancer? An epidemiologic analysis of mortality in a cohort of elderly breast cancer patients. J Gerontol A Biol Sci Med Sci 1998; 53:372-378

16. Young JL Jr, Roffers SD, Ries LAG, Fritz AG, Hurlbut AA. SEER Summary Staging Manual: Codes and Coding Instructions, National Cancer Institute, NIH Pub. No. 01-4969, Bethesda, MD. 2001

17. Chou WC, Wang F, Cheng YF, et al. A simple risk stratification model that predicts 1-year postoperative mortality rate in patients with solid-organ cancer. Cancer Med. 2015; doi: 10.1002/cam4.518.

18. Al-Refaie WB, Parsons HM, Habermann EB, et al. Operative outcomes beyond 30-day mortality: colorectal cancer surgery in oldest old. Ann Surg. 2011; 253: 947-52.

19. Lagier A, Mimouni-Benabu O, Baumstarck K, et al. The influence of age on postoperative complications after total laryngectomy or pharyngolaryngectomy. Eur J Surg Oncol. 2014; 40: 202-7.

20. Tzeng CW, Cooper AB, Vauthey JN, et al. Predictors of morbidity and mortality after hepatectomy in elderly patients: analysis of 7621 NSQIP patients. HPB (Oxford). 2014; 16: 459-68.

21. Powell HA, Tata LJ, Baldwin DR, et al. Early mortality after surgical resection for lung cancer: an analysis of the English National Lung cancer audit. Thorax. 2013; 68: 826-34

22. Iversen LH, Ingeholm P, Gögenur I, et al. Major reduction in 30-day mortality after elective colorectal cancer surgery: a nationwide population-based study in Denmark 2001-2011. Ann Surg Oncol. 2014; 21: 2267-73.

23. Chou WC, Liu KH, Lu CH, et al. To operate or not: prediction of 3-month postoperative mortality in geriatric cancer patients. J Cancer.2016; 7: 14-21.

24. Korc-Grodzicki B, Downey RJ, Shahrokni A, et al. Surgical considerations in older adults with cancer. J Clin Oncol. 2014; 32: 2647-53. 
25. Barone BB, Yeh HC, Snyder CF, et al. Long-term all-cause mortality in cancer patients with preexisting diabetes mellitus: a systematic review and meta-analysis. JAMA.2008; 300: 2754-64.

26. Yancik R, Wesley MN, Ries LA, et al. Effect of age and comorbidity in postmenopausal breast cancer patients aged 55 years and older. JAMA. 2001; 285: 885-92.

27. Wu AH, Kurian AW, Kwan ML, et al. Diabetes and other comorbidities in breast cancer survival by race/ethnicity: the California Breast Cancer Survivorship Consortium (CBCSC). Cancer Epidemiol Biomarkers Prev. 2015; 24: 361-8.

28. Piccirillo JF, Tierney RM, Costas I, et al. Prognostic importance of comorbidity in a hospital-based cancer registry. JAMA. 2004; 291: 2441-7

29. Koppie TM, Serio AM, Vickers AJ, et al. Age-adjusted Charlson comorbidity score is associated with treatment decisions and clinical outcomes for patients undergoing radical cystectomy for bladder cancer. Cancer. 2008; 112: 2384-92.

30. Ramsden K, Laskin J, Ho C. Adjuvant chemotherapy in resected stage II non-small cell lung cancer: evaluating the impact of dose intensity and time to treatment. Clin Oncol (R Coll Radiol). 2015; 27: 394-400.

31. Malietzis G, Mughal A, Currie AC, et al. Factors implicated for delay of adjuvant chemotherapy in colorectal cancer: a meta-analysis of observational studies. Ann Surg Oncol. 2015; 22: 3793-802.

32. Earle CC, Neville BA. Under use of necessary care among cancer survivors. Cancer. 2004; 101: 1712-9.

33. Snyder CF, Frick KD, Herbert RJ, et al. Quality of care for comorbid conditions during the transition to survivorship: differences between cancer survivors and noncancer controls. J Clin Oncol. 2013; 31: 1140-8.

34. Potosky AL, Han PK, Rowland J, et al. Differences between primary care physicians' and oncologists' knowledge, attitudes and practices regarding the care of cancer survivors. J Gen Intern Med. 2011; 26: 1403-10.

35. McCabe MS, Bhatia S, Oeffinger KC, et al. American Society of Clinical Oncology statement: achieving high-quality cancer survivorship care. J Clin Oncol. 2013; 31: 631-40.

36. Reed SC, Partridge AH, Nekhlyudov L. Shared medical appointments in cancer survivorship care: a review of the literature. J Oncol Pract. 2014; [Epub ahead of print].

37. Damhuis RA, Wijnhoven BP, Plaisier PW, et al. Comparison of 30-day, 90-day and in- hospital postoperative mortality for eight different cancer types. Br J Surg. 2012; 99: 1149-54.

38. Dekker JW, van den Broek CB, Bastiaannet E, et al. Importance of the first postoperative year in the prognosis of elderly colorectal cancer patients. Ann Surg Oncol. 2011; 18: 1533-9.

39. Yasmeen S, Xing G, Morris C, et al. Comorbidities and mammography use interact to explain racial/ethnic disparities in breast cancer stage at diagnosis. Cancer. 2011; 117: 3252-61. 